\title{
REFINING ANTICIPATION OF DEGRADED BONE MICROSTRUCTURES DURING OSTEOPOROSIS BASED ON STATISTICAL HOMOGENIZED RECONSTRUCTION METHOD VIA QUALITY OF CONNECTION FUNCTION
}

\author{
SEYED FARZAD FAMOURI \\ School of Mechanical Engineering, College of Engineering, University of Tehran, \\ Tehran, Iran \\ The Robotic Surgery Lab., Mechanical Engineering Dept., Concordia University, Montreal, QC, Canada \\ AMIRHOSSEIN BAGHERIAN and ARMIN SHAHMOHAMMADI \\ School of Mechanical Engineering, College of Engineering, University of Tehran, \\ Tehran, Iran \\ DANIEL GEORGE \\ University of Strasbourg ICube/CNRS, 2 rue Boussingault, 67000 Strasbourg, \\ France \\ MOSTAFA BAGHANI and MAJID BANIASSADI* \\ School of Mechanical Engineering, College of Engineering, University of Tehran, \\ Tehran, Iran \\ baghani@ut.ac.ir \\ m.baniassadi@ut.ac.ir
}

\begin{abstract}
Nowadays, osteoporosis disease that is related to aging has become a proliferating problem in worldwide society. It is therefore is crucial to understand its evolution and predict this phenomenon precisely for different types of bone and volume fractions with adequate mathematical model. The application of statistical reconstruction method would be a helpful tool to predict osteoporosis for the simplified bone microstructures. To model osteoporosis evolution over time, in a first step we propose to degrade the volume fraction with a mathematical model to reach any determined volume fraction between the initial condition and the degraded one with a statistical interpolation. In a second step, the degraded microstructure will be optimized using a statistical descriptor. The final optimized microstructures will be discussed as a function of the effective mechanical properties. The capability of quality of connection and two-point correlation functions in 3D models and their application in the optimization of reconstructed interpolated models are going to be demonstrated. Finally, we will demonstrate and discuss the advantages of using the Quality of Connection Function (QCF) as a replacement of Two-Point Correlation Function (TPCF) over the sole statistical descriptor named Two-point Correlation function. We will show that QCF descriptor is better than TPCF only to find the optimized reconstructed models in a determined volume fraction.
\end{abstract}

Keywords: Osteoporosis; Interpolation; Quality of Connection Function; Tow-Point Correlation Function; Two-phase Recovery; Compliance Matrix; Young Modulus

\section{Introduction}

Osteoporosis is one of the most worrying health issues of aging. It occurs in both cancellous and cortical bones; they become more porous, fragile and prone to fractures. Bone remodeling is dependent on its porosity and strain energy density that is known to be the significant factor of fragility [Bakalova, et al., 2018]. The degradation due to osteoporosis varies greatly between cancellous and cortical bone, and different bones, but it is typically higher in trabecular bones [Ram et al., 2006 - Lang et al 2006]. The density and distribution of trabecular bone is

\footnotetext{
* Corresponding author: Majid Baniassadi

School of Mechanical Engineering, College of Engineering, University of Tehran, Tehran 1439957131, Iran

Phone: +982161114035

Fax: +982188013029

Email: m.baniassadi@ut.ac.ir
} 
directly related to the bone loss. Moreover, this relation also depends on sex, age and type of bones [Chen et al., 2013]. Bone remodeling behavior affects the development of osteoporosis procedure. Hence, the mechanobiological relations linked to the mechanical behavior of the tissue, gaining stiffness with a minimum amount of material, have become a focus of researchers in optimization problems [Carter et al., 1996-Casanova et al. 2010- Huiskes et al., 2000].

When it comes to the effect of the material microstructures in continuum models that are widely discussed in the prior research [Lekszycki,2002-Lekszycki,2005, Pivonka et al.,2008-Scala et al.,2017-Madeo et al.,2012], continuum theory falls short to describe the correct material behavior. Continuity of the material is not realistic any more where scaling is not integrated into the structural evolution due to its heterogeneous attributes at the small scales; this concept was raised by Cauchy continuum theory [Hegedus et al., 1976-Andreaus et al., 2011Prendergast et al., 1994-Doblaré et al., 2002-Dell'Isola, F., et al., 2015-Misra et al., 2015-Placidi et al., 2015Abali et al., 2017-Dell'Isola, F., et al., 2017]. Homogenization methods like two-phase recovery reconstruction [Rémond et al., 2016-Martin et al., 2017-George et al., 2017-Spingarn et al., 2017-Kazempour et al., 2019Hashemi et al.2019] can be used to address this issue.

Horvath and Chiba [Horvath, 2016-, Chiba et al., 2013] consider bone material as a complex heterogeneous microstructure which is formed on organic (collagen and proteins) and inorganic (bone mineral) chemicals. Although, bone is usually assumed as a poroelastic material consisting of a solid phase, an interstitial fluid phase and a solute phase, that affect each the mechanical loading regimes [Ghimire et al., 2018], the analysis of the changes in bone structures such as osteoporosis can be done, on a first approximation, using an elastic porous material [Kazempour et al., 2019] from the mechanical point of view. This approach is supported by the fact that although fluid phase plays a role in the overall mechanical support of the structure, it is not the first order parameter (as this comes mainly from the bone stiffness itself), and second, fluid compressibility will not change much between two degradation state of osteoporosis, which therefore will not affect much the bone microstructure evolution.

The most appropriate method for a representation of the heterogeneous materials properties are known to be statistical descriptions, which are widely used in reconstruction and homogenization [Torquato et al.,2002Baniassadi et al.,2011-Hasanabadi et al.2016] of these microstructures, based on correlation functions [Izadi et al.,2017- Fullwood et al.,2008]. In two of our most recent research, the influence of the local bone microstructure distribution on the macroscopic bone density evolution during a long period time has been analyzed through the local strain energy distribution and two-point correlation functions reconstruction [Bagherian et al.,2019Sheidaei et al,2019].

[Tawara et al., 2019] developed a computational framework with multi-scale stress analyses and remodeling simulations. Their findings showed that fracture risk could be distinguished in more detail by the time-dependent variation of bone quality. They showed that the relationship between the present percentage of the high stress and the decrease of bone density was not proportional in the osteoporotic reconstruction process, however, it increased more when the bone mass was reduced. In 2020, [Kraiem et al., 2020] linked the FEM with a micromechanical law, proposing to present an exhaustive description of the human bone mechanical behavior.

The main outcome of this work is the prediction of the osteoporosis procedure through statistical approaches. The first one is known generally, as Two-point correlation function and the newest one called Quality of Connection Function. The changes in the structures with high efficiency using these two functions are studied during the osteoporosis process. The methodology is based on statistical mechanics that could be especially useful when two stages in a system are chose from one region not exactly one point. Through our proposed system, the statistical procedure of changes in stages could be predicted.

\section{Procedure}

In this study, the Initial bone microstructure is modeled based on X-ray micro-computed tomography images. It is then degraded through our previous osteoporosis model [Bagherian et al., 2019]. Next, by statistical reconstruction, microstructures are interpolated with statistical reconstruction. The microstructures with the closest mechanical properties and the same volume fraction as real bone are detected through statistical descriptors. Finally, we estimate the mechanical properties of these reconstructed microstructures with Finite element method that can estimate the effective elasticity tensor of a composite [Andreassen et al., 2014]. By 
estimating the mechanical properties, we can compare the capability and accuracy of the new descriptor named Quality of Connection function to obtain accurate results compared to a two-point correlation function.

\subsection{Modeling Degradation for the Initial Bone Sample}

The FE bone samples were obtained from real bone femur microstructures extracted from an 85-year-old woman. The experimental procedure used to obtain the sample is detailed in Sheidaei et al. [Andreassen et al., 2014]. Simulation of bone degradation in this study is performed by using the recent bone degradation model developed by Bagherian et al. [Bagherian et al., 2019]. According to this pixel-based model, the bone degradation occurs only at the surface of the bone, in which bone voxels are directly in contact with the bone marrow voxels. Models are constructed based on micro-CT images of trabecular bone samples. Each pixel represents a voxel in the finite element model and its property assigned according to the corresponding Hounsfield value. The bone remodeling occurs at the surface of the bone from degradation equations that are applied to the bone voxels facing the bone marrow voxels in their neighborhood. The voxels that are not connected to bone marrow remain unchanged. The degradation rate is not equal among all surfaces of the bone voxels. The rate of degradation is a function of the amount of contact surface with the bone marrow. Therefore, the bone microstructure degrades non-uniformly. It should be noticed that degradation might cause loss of connections between voxels which affects the bone strength.

According to the aforementioned degradation method, bone degeneration was simulated on a trabecular bone microstructure with 47.1 percent volume fraction at the initial step. The microstructure was composed of three clusters, in which volume fraction of the main cluster was 47.09 percent which is the focus of our study. Microstructure degraded in multiple steps and volume fraction of the bone sample decreased to 23.49 percent in the final step. The initial and degraded models, as with using the degradation model of Bagherian et al. [Bagherian et al., 2019], are showed in Fig. 1.
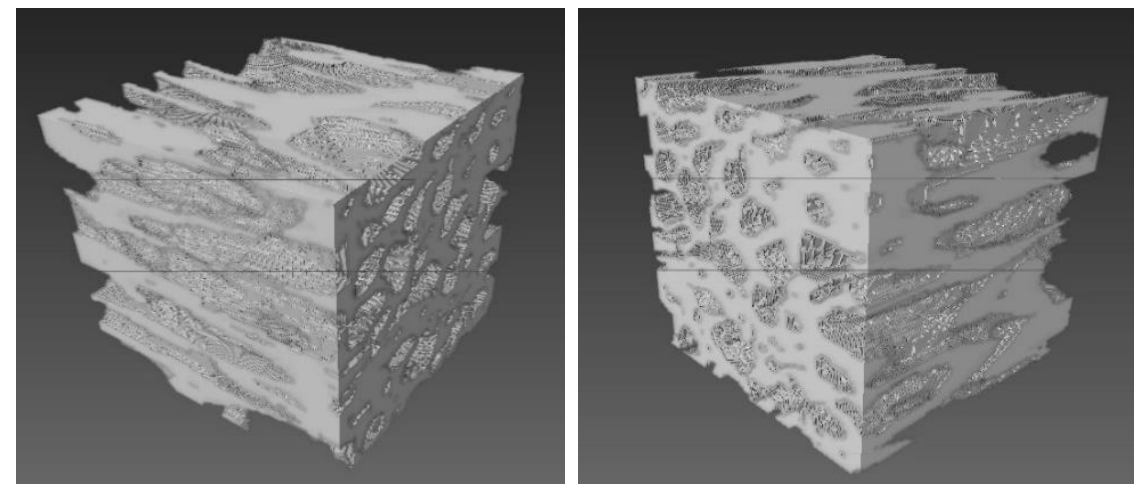

(a)
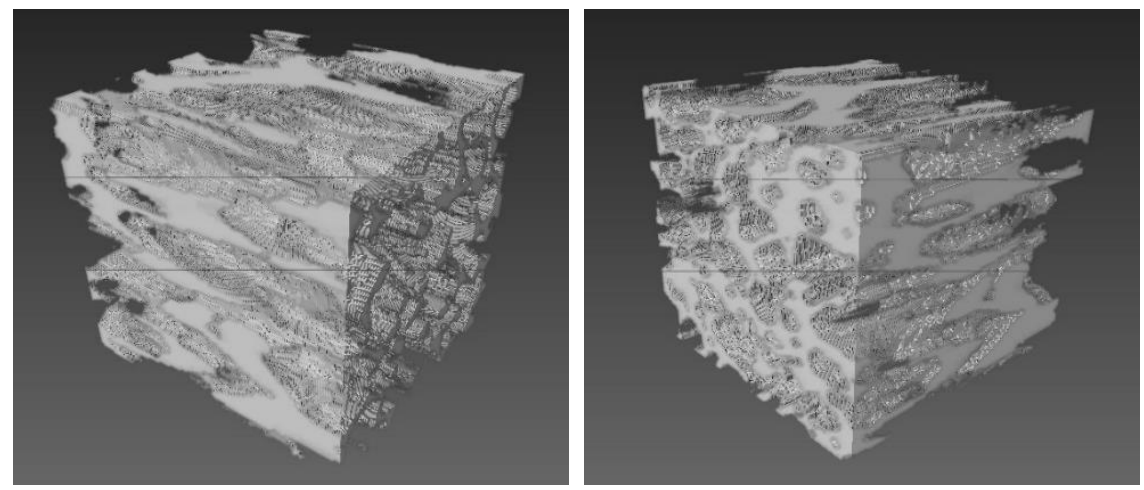

(b)

Fig. 1. Representation of modeling osteoporosis in two angles. (a) Microstructure of bone with volume fraction of 47.1\%. (b): Degraded microstructure with volume fraction of $23.49 \%$ 


\subsection{Statistical Interpolation}

\subsubsection{Two-Point Correlation Function}

Statistical Reconstruction method is the understanding of a microstructure from its statistical correlation function that is obtained from SEM images. In this study, by employing an approximation of full-spectrum two-point correlation functions (TPCF) of a 3D microstructure, a reconstruction has been performed. In this study, phaserecovery algorithms are used to reconstruct 3D-microstructure from 2D images of the bone. [Torquato et al., 2002- Andreassen et al.,2014 37] Discretizing grid points of the Eigen 2D images is the starting point in the phase recovery algorithm. This distribution is designated by $x_{p}^{q}$ and the characteristic function defined by Eq. (2.1), where the superscript $q$ identifies the phase number and $p$ identifies the number of each grid that defines the $2 \mathrm{D}$ images. Eq. (2.1) the amount is equal to one when $\mathrm{p}$ is in phase q otherwise it would be equal to zero.

$$
x_{p}^{q}=\left\{\begin{array}{l}
1 \\
0
\end{array}\right.
$$

In our two-phase models, the phases are named $\mathrm{q}$ and $q^{\prime}$ and also, the grid point is specified with a number between 0 and p-1. According to Eq. 1, one point-correlation function for phase q is defined in Eq. (2.2).

$$
c_{1}^{q}=\left\langle x_{p}^{q}\right\rangle=\frac{1}{p} \sum_{p=0}^{p-1} x_{p}^{q}
$$

Eq. (2.2) is an ensemble average, and the asymptotic value for the above equation is almost equivalent to the volume fraction for each phase. By applying one-point correlation functions, the upper and lower bound properties of heterogeneous and limited microstructure can be found [60].

The two-point correlation function, which is the first descriptor that can acquire geometrical specifications of heterogeneous materials, is defined below as:

$$
c_{2}^{q q^{\prime}}(s)=\frac{1}{p} \sum_{p=0}^{p-1} x_{p}^{q} x_{p+s}^{q}
$$

It`s concept for each vector is illustrated in different color as Fig. 2 below:

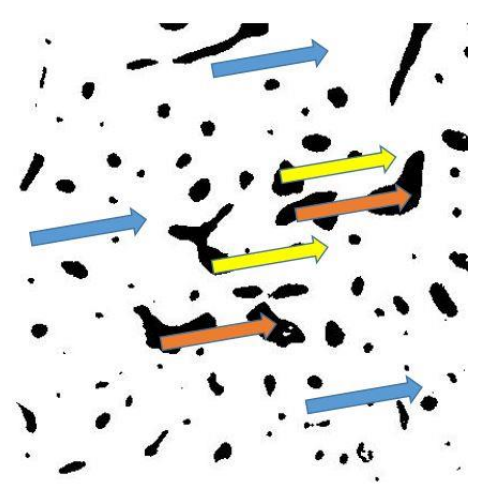

Fig. 2. Schematic of Two-point correlation function for a two-phase cortex bone microstructure.

In the Eq. (2.3), $\mathrm{s}$ is the correlation vector, which is defined as the relative difference between the position vectors of the voxels.

TPCFs in a two-phase model have the relations below:

$$
\begin{gathered}
c_{2}^{q q}+c_{2}^{q q^{q}}+c_{2}^{q q q}+c_{2}^{q q^{q}}=1 \\
c_{2}^{q q^{q}}=c_{2}^{q q q} \\
c_{2}^{q q^{q}}(s)=\frac{1}{p} \sum_{p=0}^{p-1} x_{p}^{q} x_{p+s}^{q}
\end{gathered}
$$


In Eq. (2.6), $v_{n}$ is the volume fraction of phase $\mathrm{q}$, which has a constant value. In consequence, there is only one independent TPCF for any two-phase microstructure.

Constraints of Eq. (2.3) are provided below.

$$
\begin{gathered}
\lim _{s \rightarrow 0} c_{2}^{n m}(s)=\left\{\begin{array}{l}
v_{n} \text { if }, n=m \\
0 \text { if }, n \neq m
\end{array} n, m \in\left\{q, q^{\prime}\right\}\right. \\
x_{k}^{q}=F\left(x_{p}^{q}\right)=\frac{1}{p} \sum_{p=0}^{p-1} x_{p}^{q} e^{\left(\frac{2 \pi i p k}{q}\right)}=\frac{1}{p}\left|X_{k}^{q} \| e^{i \theta_{k}^{q}}\right|
\end{gathered}
$$

The Fast Fourier Transform of the microstructure is:

$$
\lim _{s \rightarrow \infty} c_{2}^{n m}(s)=\left\{\begin{array}{l}
v^{2}{ }^{2} \text { if }, n=m \\
v_{n}{ }^{v}{ }_{m} \text { if }, n \neq m
\end{array}, n, m \in\{q, q\}\right.
$$

Where, $\left|X_{k}^{q}\right|$ and $\theta_{k}^{q}$ are the amplitude and the phase of the Fourier transform, respectively. Fulwood improved Eq. (2.9) by using TPCF instead of microstructure function [63].

$$
c_{k}^{q q^{\prime}}=F\left(c_{2}^{q q^{\prime}}(s)\right)=\frac{1}{p} \sum_{p=0}^{p-1} x_{p}^{q} e^{\left(\frac{2 \pi i p k}{q}\right)}=\frac{1}{p}\left|X_{k}^{q}\right| e^{i \theta_{k}^{q}}\left|X_{k}^{q}\right| e^{-i \theta_{k}^{q}}
$$

When $q=q^{\prime}$, TPCFs for every microstructure can be calculated with:

$$
c_{k}^{q q}=\frac{1}{p}\left|X{ }_{k}^{q}\right|^{2}=\frac{1}{p} \tilde{X}_{k}^{q} X_{k}^{q}
$$

The auto-covariance function is defined for the vector and $\mathrm{s}$ is used for representing bone microstructures. It is a linear and scaled function for TPCF, ranging between 0 and 1 when $s$ varies between $s=0$ and $s=\infty$, in order.

$$
f_{2}^{n}(s)=\frac{c_{2}^{q q}(r)-v_{q}^{2}}{v_{q}\left(1-v_{q}\right)}
$$

A combination of two or more auto-covariance functions for statistically homogeneous models satisfies the essential condition of Eq. (2.7) and Eq. (2.8).

\subsubsection{Quality of Connection Function}

Two-point correlation functions are not precisely detecting the connection of phases in the 2D and 3D models, so by applying the novel descriptor we are looking to improve that. Before introducing the new descriptor called Quality of Connections, two concepts should be defined. The first is the shortest distance (SD) and the second is the shortest path (SP) between two pixels or voxels of the same state in 2D and 3D microstructures. They are illustrated in the Fig.3.

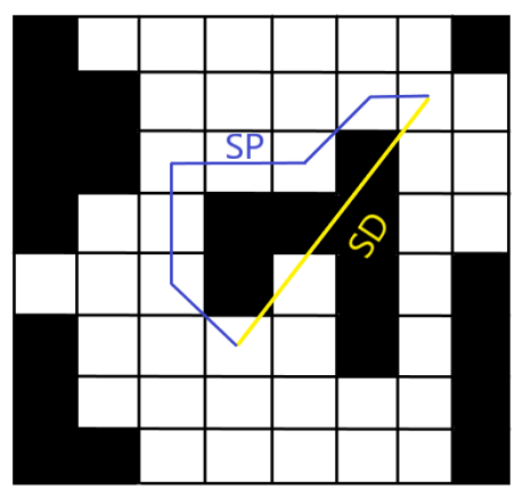

Fig. 3. Presentation of shortest distance and shortest path between two initial and target nodes in a two-phase microstructure 
The SP is the length of the path between initial and target voxels crossing through the same phase and calculated by the A-Star algorithm, in which a cost function is defined for every path between two determined nodes. As a result, we obtain a list of all possible routes, and the path with the smallest cost is selected as SP. Voxels from other phases are assumed as obstacles. Furthermore, this algorithm detects routes that are not in dead-end directions.

The value derived from SD / SP is always between zero and one. When the fraction comes close to one, it means that the shortest distance is almost equal to the shortest path, corresponding to a good connection between two pixels. Conversely, when the ratio is close to zero, it corresponds to a poor connection between pixels.

QCF is defined as the ratio between the total SD and the total SP from a large number of similar vectors generated in a given 2D or 3D microstructure. If the initial and target nodes are located in different clusters, there is no path between the nodes. In that situation, SP is assumed infinite so the value of the fraction is equal to zero. This statistical descriptor is capable to determine the quality of the connections between different degradation states and helps to have detailed statistical information about the evolution of the bone microstructures.

\subsubsection{Blind Searching}

The auto-covariance of the combined microstructure is defined as.

$$
f_{2}^{q}(r)=\alpha f_{2}^{q^{1}}+(1-\alpha) f_{2}^{q^{2}}
$$

Where $f_{2}^{q 1}$ and $f_{2}^{q 2}$ are two different auto covariance functions linked to the first and last measurement point of the osteoporosis process. $\alpha$ is considered as a constant. The algorithm looks for an optimized value of $\alpha$, giving the desired mechanical properties for the microstructure, comparing with bone models with the same volume fraction. Eq. (2.13) satisfies conditions (2.7) and (2.8) only when $\alpha$ is in the range between 0 and 1 . The procedure is summarized below.

1. First $\alpha$ is chosen any constant number between 0 and 1 .

2. As a result, $f_{2}^{q}(r)$ is calculated via merging the initial bone microstructure and degraded microstructure TPCFs.

3. A new TPCF is found from the inverse of Eq. (2.12) and then it is used as input data of step.4

4. The microstructure is reconstructed with a two-phase recovery method (2.2.4).

5. The smallest error is found and compared with bone models with the same volume fraction.

6. Consequently, the optimum $\alpha$ is found.

\subsubsection{Two-Phase Recovery Method for Homogenized Reconstruction}

Reconstruction procedure means producing a microstructure based on its assigned statistical correlation functions. The inputs of this method are the TPCFs based on Eq. (2.12) and (2.13). The phase recovery algorithm in this study is found on Eq. (2.11). The amplitudes of the microstructure functions are computed trough inserting a set of TPCFs into this equation. Toward obtaining the microstructure correlation functions completely, recovering the phases aforementioned in Eq. (2.9) by phase recovery algorithm is sufficient, so finding $x_{p}^{q}$ for all points given the full set of TPCFs would be the algorithm objective.

The four steps of the two-phase recovery algorithm, highlighted in blue in the flowchart, are defined in Fig.4.

1. A primary random microstructure is generated ( $\left.x_{p \text { ite }}^{q}\right)$ for the first iteration. Then it's Fast Fourier Series of microstructure function is calculated ( $x_{k \text { ite }}^{q}$ ) through Eq. 9.

2. The phases of the FFS are preserved but their modulus $\left(\left|x_{p \text { ite }}^{q}\right|\right)$ is replaced with the square root of the $c_{k \text { ref }}^{q q}$ multiplied by $q$ or $\left|x_{p \text { ref }}^{q}\right|$ using Eq. 11.

3. The inverse Fourier transform of $\left|X_{k \text { ref }}^{q}\right| e^{i \theta_{k i t e}^{q}}$ is taken from the Eq. 9 and then it is considered to be new $x_{p \text { ite }}^{q}$.

4. After applying constraints in real space to the obtained $x_{p}^{q}$ ite by rounding its value, the realized microstructure in this step is used as an input for the primary step.

The error between the TPCFs of the reconstructed microstructure and $c_{2 \text { ref }}^{q q}$ is calculated based on Eq. (2.14). 


$$
\text { Error }=\frac{1}{p} \sum_{s=0}^{s=p-1} \frac{\left|c_{2}^{q q}(s)_{i t e}-c_{2}^{q q}(s)_{r e f}\right|}{c_{2}^{q q}(s)_{r e f}}
$$

If the convergence criteria are satisfied the algorithm will be stopped and the $x_{p \text { ite }}^{q}$ of step four would be considered as the final reconstructed microstructure [54]. The flowchart of this algorithm is shown in the fig.4.

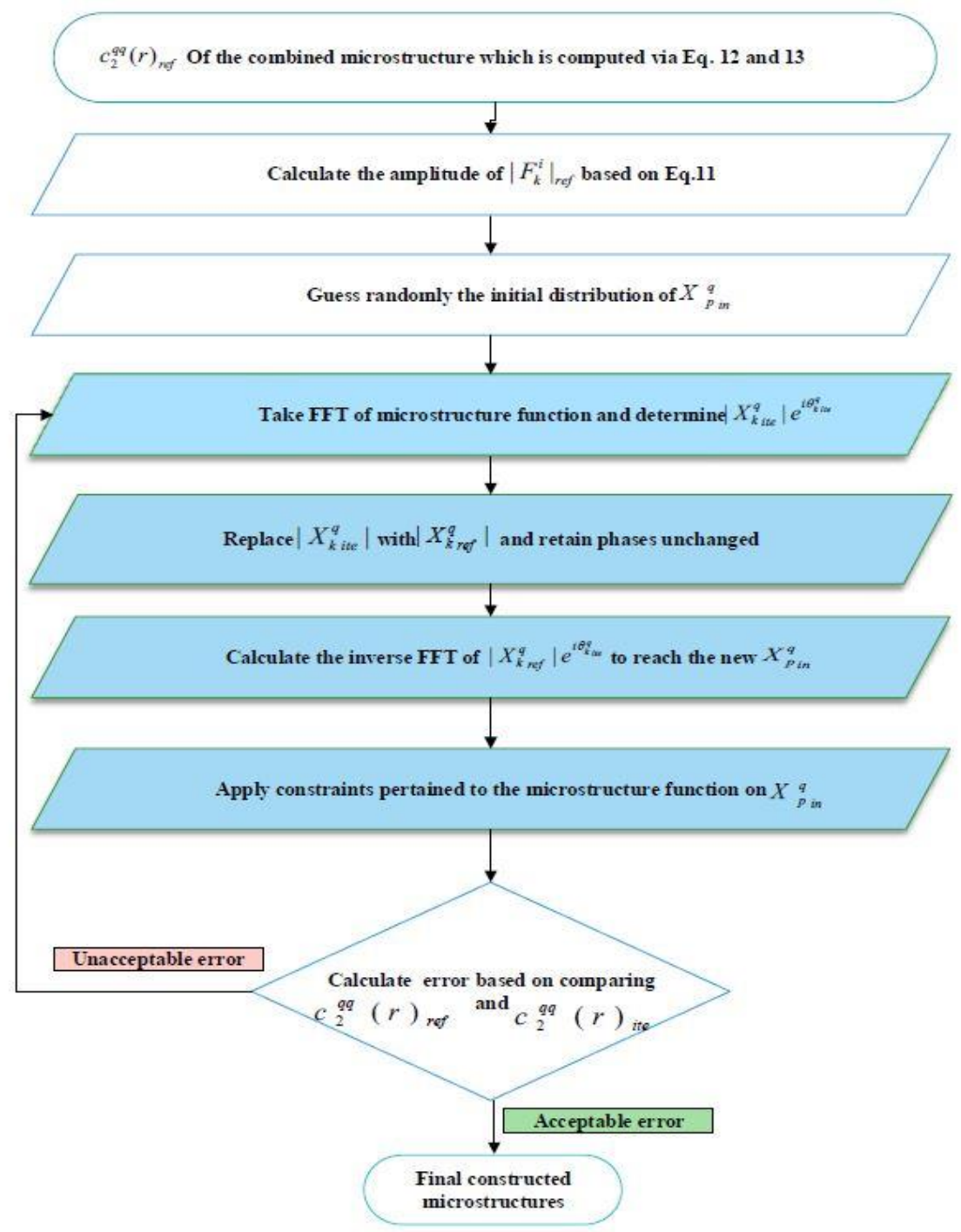

Fig. 4. Phase recovery algorithm

\subsection{Characterization}

The microstructure of composite or heterogeneous materials can often be described by a unit cell, which is periodically repeated in one or more directions. Bone microstructure was considered in such a way [Andreassen et al., 2014] as it provides an easier description for mechanical properties homogenization.

The macroscopic elasticity tensor $\left(E_{i j k l}^{H}\right)$ of a composite material based on the theory of homogenization, is computed as the following.

$$
E_{i j k l}^{H}=\frac{1}{|V|} \int_{V} E_{p q r s}\left(\varepsilon_{p q}^{0(i j)}-\varepsilon_{p q}^{(i j)}\right)\left(\varepsilon_{r s}^{0(k l)}-\varepsilon_{r s}^{(k l)}\right) d V
$$

In this equation, $E_{p q r s}$ is defined as the locally varying stiffness tensor and $\varepsilon_{p q}^{0(i j)}$ are designated macroscopic strain fields (in 2D structures, there are $\varepsilon_{1}, \varepsilon_{2}$ and $\gamma_{12}$ ). $|V|$ is the volume of the unit cell. The elasticity equation is defined by the Eq. (2.16). 


$$
\int_{V} E_{i j p q} \varepsilon_{i j}(v) \varepsilon_{p q}\left(X^{k l}\right) d V=\int_{V} E_{i j p q} \varepsilon_{i j}(v) \varepsilon_{p q}^{o(k l)} d V, \forall v \in V
$$

Where, $v$ is a virtual displacement field. By solving Eq.16, $\chi^{k l}$ is calculated and also, the locally varying strain fields are defined as:

$$
\varepsilon_{p q}^{(i j)}=\varepsilon_{p q}\left(X{ }^{i j}\right)=\frac{1}{2}(X \underset{p, q}{i j}+X \underset{q, p}{i j})
$$

Eq. (2.15) can be solved by using the finite element method. The stiffness matrix is defined as:

$$
K=\sum_{e=1}^{N} \int_{v_{e}} B_{e}^{T} C_{e} B_{e} d V_{e}
$$

Where, $B_{e}$ is the strain-displacement of the element, $\mathrm{N}$ is the number of elements and $V_{e}$ is the volume of each element. Also, $C_{e}$ for the isotropic composite is determined in the Eq. (2.18).

$$
C_{e}=\lambda_{e} \cdot\left(\begin{array}{lll}
1 & 1 & 0 \\
1 & 1 & 0 \\
0 & 0 & 0
\end{array}\right)+\mu_{e} \cdot\left(\begin{array}{lll}
2 & 0 & 0 \\
0 & 2 & 0 \\
0 & 0 & 1
\end{array}\right)
$$

Moreover, $\lambda_{e}$ and $\mu_{e}$ are calculated for each element as the following.

$$
\begin{array}{r}
\mu=\frac{E}{2(1+v)} \\
\lambda=\frac{v E}{(1+v)(1-2 v)}
\end{array}
$$

For plane stress, the Eq. (2.22) is used.

$$
\hat{\lambda}=\frac{2 \mu \lambda}{\lambda+2 \mu}
$$

The stiffness matrix $(\mathrm{K})$ is divided into 2 corresponding parts:

$$
K=\sum_{e=1}^{N} K_{e}=\sum_{e=1}^{N} \lambda_{e} K_{\lambda}+\mu_{e} K_{\mu}
$$

The right-hand side of Eq. (2.16) is called loads $\left(f^{i}\right)$ :

$$
f^{i}=\sum_{e} \int_{v_{e}} B_{e}^{T} C_{e} \varepsilon^{i} d V_{e}
$$

Where,

$$
\varepsilon^{1}=\left\{\begin{array}{l}
1\rceil \\
0 \\
0 \\
0
\end{array}\right\rfloor, \varepsilon^{2}=\left[\begin{array}{l}
0\rceil \\
1 \\
\vdots \\
0
\end{array}\right\rfloor, \varepsilon^{3}=\left[\begin{array}{l}
\lceil 0\rceil \\
0 \\
\vdots
\end{array}\right\rfloor
$$

Eq. (2.26) is obtained via accumulating loads as blow:

$$
f^{i}=\sum_{e=1}^{N} \lambda_{e} f_{\lambda}^{i}+\mu_{e} f_{\mu}^{i}
$$

Eventually, the displacement vectors $\chi^{i}$ are computed and also, Eq. (2.27) is obtained:

$$
k X^{i}=f^{i}
$$


By obtaining displacement fields, the homogenized constitutive matrix $\mathrm{C}^{\mathrm{H}}$ can be estimated from Eq. (2.28).

$$
C_{i j}^{H}=\frac{1}{|V|} \sum_{e=1}^{N} \int_{V_{e}}\left(X_{e}^{0(i)}-X_{e}^{(i)}\right) K_{e}\left(X_{e}^{0(j)}-X_{e}^{(j)}\right) d V_{e}
$$

\section{Results and Discussion}

To better understand the necessity of reconstruction, we have generated random microstructures with the same volume fraction of the reconstructed microstructures. In the first stage, the difference between random matrices and reconstructed bone microstructures will be discussed. Then, the best matrix for each volume fractions will be chosen through TPCF and QCF methods. In the last step, the superiority of the QCF method over TPCF will be shown through computing the effective elastic modulus of microstructures.

\subsection{Random Matrices and Comparison}

First, random microstructures with volume fractions of $0.4398,0.4176$, and 0.3149 are generated with MATLAB. Their 3D models having voxel shape are demonstrated below in the Fig.5. In this figure, each cluster is distinguished by a color.

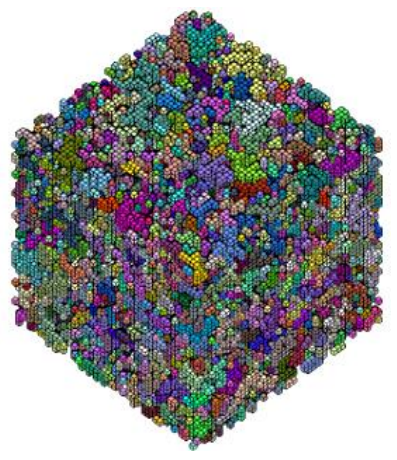

(a)

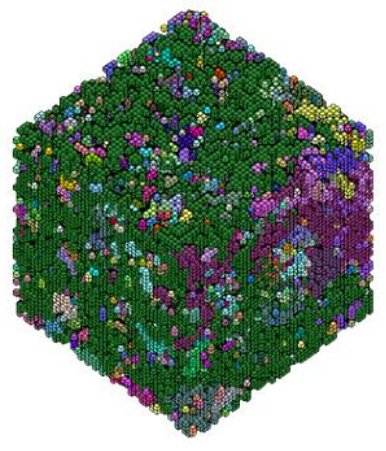

(b)

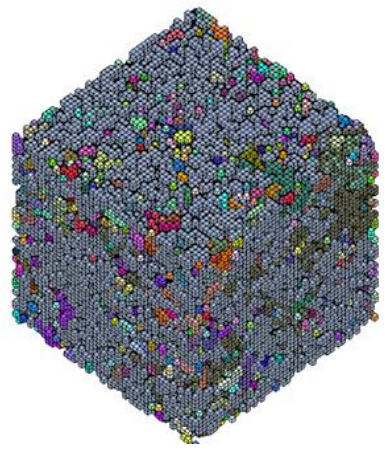

(c)

Fig. 5. Randomly generated microstructures with volume fraction of (a) $31.49 \%$, (b) $41.76 \%$, (c) $43.98 \%$

In the reconstructed model, the bone particles get connected and form a cluster that is called the percolation threshold effect. When a random microstructure is generated this phenomenon doesn't happen. In the low volume fraction samples, the number of clusters is higher than the ones with higher volume fraction due to the lack of connections. The main cluster in the $44 \%$ figure is dark blue and contains more voxels than the dark green one in $41 \%$ model. In addition, these two clusters have percolation in three main directions. In contrast, the cluster having percolation in all directions can hardly be found in the $31 \%$ model. By comparing the random models with the real ones, it is clear that they do not resemble bone`s morphology and the percolation threshold effect does not happen.

The TPCF of their matrices is calculated based on Eq. (2.9)-(2.11) and their compared Errors with real bone are shown in Table 1.

Table 1. TPCF differences between random matrices and bone models with the same volume fraction.

\begin{tabular}{rc}
\hline Random Model Volume Fraction & TPCF Differences \\
\hline $\mathbf{3 1 \%}$ & $19.11 \%$ \\
\hline $\mathbf{4 1 \%}$ & $11.12 \%$ \\
\hline $\mathbf{4 4 \%}$ & $9.833 \%$ \\
\hline
\end{tabular}

Errors related to the reconstructed models are remarkably lower than random ones (see section 3.2). Errors decrease by increasing the volume fraction. With lower volume fraction, the error increases because of the distinction in their clusters' connection. 
Despite the differences pertained to their morphology and their TPCF, the random matrices have a distinct Young modulus in both $(1,0,0)$ and $(0,1,0)$ directions. The effective compliance matrix of the random matrix with $41 \%$ volume fraction based on section 2.4 is found below.

$$
\left[\begin{array}{cccccc}
0.3110 * e-09 & -0.0685 * e-09 & -0.0686 * e-09 & 0.0011 * e-09 & 0.0001 * e-09 & 0.0006 * e-09 \\
-0.0685 * e-09 & 0.3095 * e-09 & -0.0685 * e-09 & 0.0002 * e-09 & -0.0006 * e-09 & -0.0005 * e-09 \\
-0.0686 * e-09 & -0.0685 * e-09 & 0.3098 * e-09 & -0.0003 * e-09 & 0.0011 * e-09 & 0.0010 * e-09 \\
0.0011 * e-09 & 0.0002 * e-09 & -0.0003 * e-09 & 0.8145 * e-09 & 0.0010 * e-09 & 0.0008 * e-09 \\
0.0001 * e-09 & -0.0006 * e-09 & 0.0011 * e-09 & 0.0010 * e-09 & 0.8111 * e-09 & -0.0008 * e-09 \\
0.0006 * e-09 & -0.0005 * e-09 & 0.0010 * e-09 & 0.0008 * e-09 & -0.0008 * e-09 & 0.8157 * e-09
\end{array}\right]
$$

The inverse of arrays [1, 1] and [2,2] in the effective compliance matrix are equal to the Young modulus in [1, $0,0]$ and $[0,1,0]$ directions, respectively. Hence, they are 3.215 and $3.231 \mathrm{GPa}$ in order. Bone structures are stronger in direction one (It is calculated in section 3.2). The closeness of Young modulus in both direction of random models relies on their homogenization of geometries shown in the figures, in all directions. Their Young modulus is provided in the Table 2 .

Table 2. Young modulus of random models in two different directions.

\begin{tabular}{ccc}
\hline Random Models & Young Modulus (1, 0, 0) GPa & Young Modulus (0, 1, 0) GPa \\
\hline $\mathbf{3 1 \%}$ & 1.383 & 1.398 \\
\hline $\mathbf{4 1 \%}$ & 3.215 & 3.231 \\
\hline $\mathbf{4 4 \%}$ & 3.683 & 3.671 \\
\hline
\end{tabular}

\subsection{Interpolation}

In this part, the $\alpha$ parameter presented in the two-phase recovery method (section 2.2.4) will be optimized through for each volume fraction and compared to the average error of TPCF and QCF of 2D sections. Next, the capability of QCF will be discussed.

\subsubsection{Two-Point Correlation Function}

After the reconstruction of the SEM images, the TPCFs of the best-reconstructed microstructures (Step four of the Two-phase recovery method) for each $\alpha$ is compared with the TPCF of the bone microstructure with the same volume fraction (Steps 5 and 6 of Blind Searching.)

Then, the best $\alpha$ will be chosen for each specific volume fractions. The error index for each reconstructed microstructure with a different value of $\alpha$ is estimated by the Eq. (3.1).

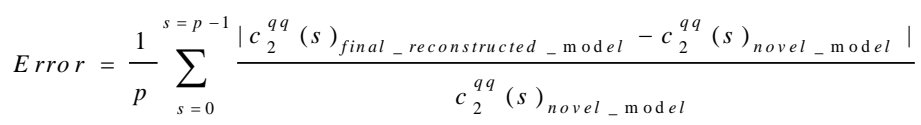

To make the calculation more accurate, the value of $\mathrm{p}$ is chosen so the range between the two TPCF disappears and the two lines overlap. This range is illustrated in Fig. 6.

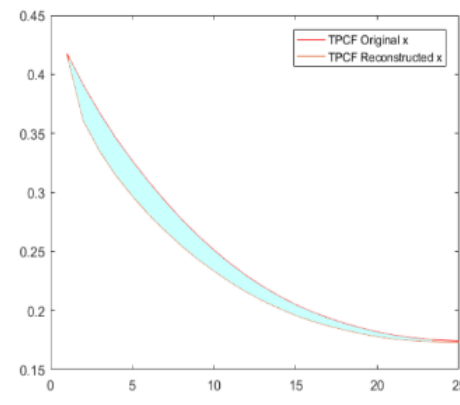

(a)

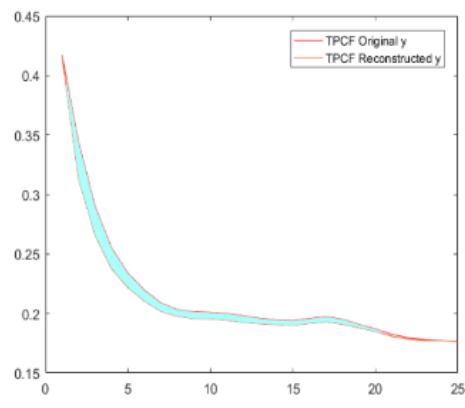

(b)

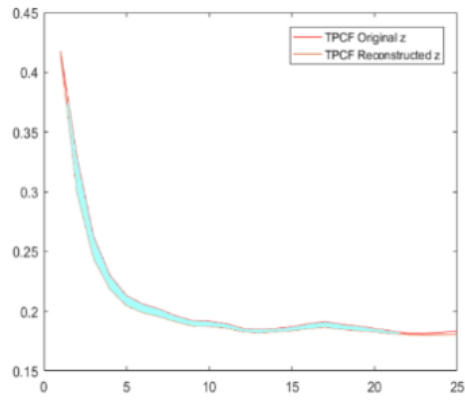

(c)

Fig. 6. Difference between the amount of TPCFs of original and reconstructed model in: a) $\mathrm{x}$ direction, b) y direction, c) $\mathrm{z}$ direction

To satisfy the conditions related to Eq. (2.7) and (2.8), $\alpha$ should be chosen between 0 and 1 . For each volume fraction, it is considered to be 0.1 at the outset and its counterpart in Eq. (2.13) would be 0.9 in the result. In the 
next step, the microstructure will be produced via the phase recovery method. Then, the TPCF error according to Eq. (2.15) would be calculated. For the next iteration, $\alpha$ is increased to 0.2 and all the aforesaid procedure is repeated. This process is continued for ten steps to reach the value of 1 to find the least differentiated of statistical microstructures and the degraded microstructures. This procedure for microstructure with volume fraction of $41 \%$ is shown in the Table 3.

Table 3. TPCF error between the reconstructed sample and the real experimental bone with the same volume fraction.

\begin{tabular}{ccc}
\hline $\boldsymbol{\alpha}$ & Result's errors & Rank \\
\hline $\mathbf{0 . 1}$ & $0.7644 \%$ & 3 \\
\hline $\mathbf{0 . 2}$ & $0.7879 \%$ & 4 \\
\hline $\mathbf{0 . 3}$ & $0.3031 \%$ & 2 \\
\hline $\mathbf{0 . 4}$ & $0.1631 \%$ & 1 \\
\hline $\mathbf{0 . 5}$ & $1.065 \%$ & 6 \\
\hline $\mathbf{0 . 6}$ & $0.794 \%$ & 5 \\
\hline $\mathbf{0 . 7}$ & $1.699 \%$ & 7 \\
\hline $\mathbf{0 . 8}$ & $2.224 \%$ & 8 \\
\hline $\mathbf{1 . 0}$ & $2.423 \%$ & 9 \\
\hline & $2.931 \%$ & 10 \\
\hline
\end{tabular}

According to the Table. 1 and Table 3, the significant difference of errors from TPCF of reconstructed models and random matrices are observed.

The table below presents the optimized $\alpha$ with the lowest errors for each specific volume fraction.

Table 4. Optimized alpha coefficients for each volume fraction via TPCF method.

\begin{tabular}{ccc}
\hline Sample Volume Fractior & \multicolumn{2}{c}{$\boldsymbol{\alpha}$ Error in percen } \\
\hline $\mathbf{3 1 \%}$ & 0.2 & 0.1775 \\
\hline $\mathbf{4 1 \%}$ & 0.4 & 0.1631 \\
\hline $\mathbf{4 4 \%}$ & 0.1 & 0.2175 \\
\hline
\end{tabular}

Regarding the use of Two-point correlation function as presented above for this type of problem (i.e. quality of the microstructure reconstruction and phase connectivity), it correlates merely the state of starting and ending points of the correlation vector. In order to obtain a more precise model using TPCF, the numerical range of coefficient can be divided into smaller intervals, but the connection between phase clusters is not considered in the calculation. Therefore, higher difference in the mechanical characterization of structures using TPCF lead to larger errors. Hence, other descriptors like Two-point Cluster function, Lineal Path and Quality of Connection are needed to improve the connections and to obtain better results.

In the following, we will use the Quality of Connection Function (QCF) to improve on the results prediction.

\subsubsection{Quality of Connection Function}

To evaluate the quality of the connections in the 3D samples, the following steps are taken.

1. Vectors quality connections with a size range between 1 and maximum width of the image which is 50 in two main directions, vertical and horizontal for each 2D sections are measured. QCF presents a plot as an output, in which horizontal and vertical axes represent the length of vectors and values of quality of connections, respectively. Their figures are presented below in Fig. 7 for an example.
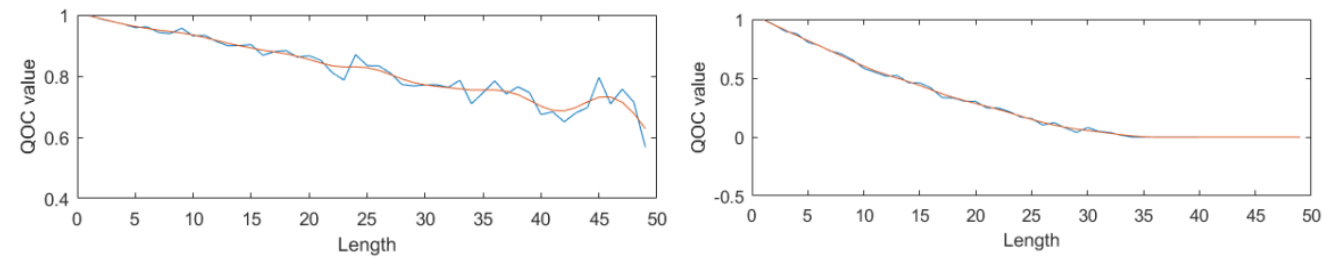

Fig. 7. Quality of connection value in both vertical and horizontal directions. Horizontal and vertical axes describe length of vectors and values of QCF, respectively. 
In our study, we have 50 images of the microstructure, while each image has $50 * 50$ pixels. As the consecutive images resemble each other closely, for our study, every third images are chosen in the three main directions to reduce the calculation. The resemblance of three consecutive microstructures with $47.1 \%$ volume fraction is descripted in Fig. 8.
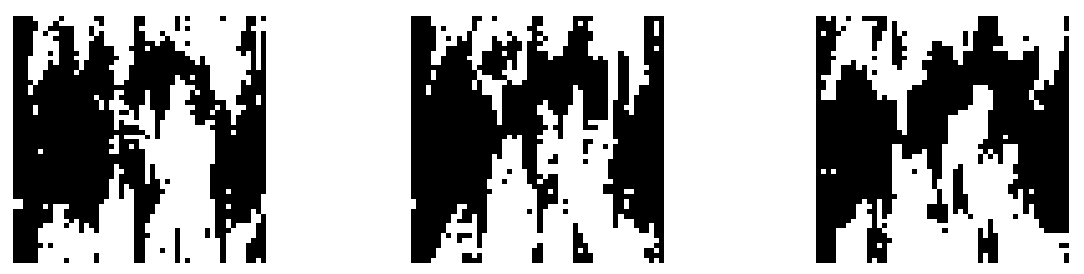

Fig. 8. Three consecutive CT images of reconstructed microstructure.

2. Since we have a determined number of vectors distributed randomly in the microstructures, the quality of connection functions was calculated two times for each case in both directions in order to check the possibility of a discrepancy. At first, the number of vectors was assumed 100 and the repeatability was checked. It is shown that the error margin was too high and the number of vectors was not high enough so the number was gradually increased. By considering 500 vectors, the random distribution of vectors showed small error and small discrepancy in QCF values. As far as the root mean square error is less than two percent in the QCF, the compatibility of the results demonstrates that the number of vectors generated in the microstructure is sufficient. The aforementioned explanation is shown in the Fig. 9.
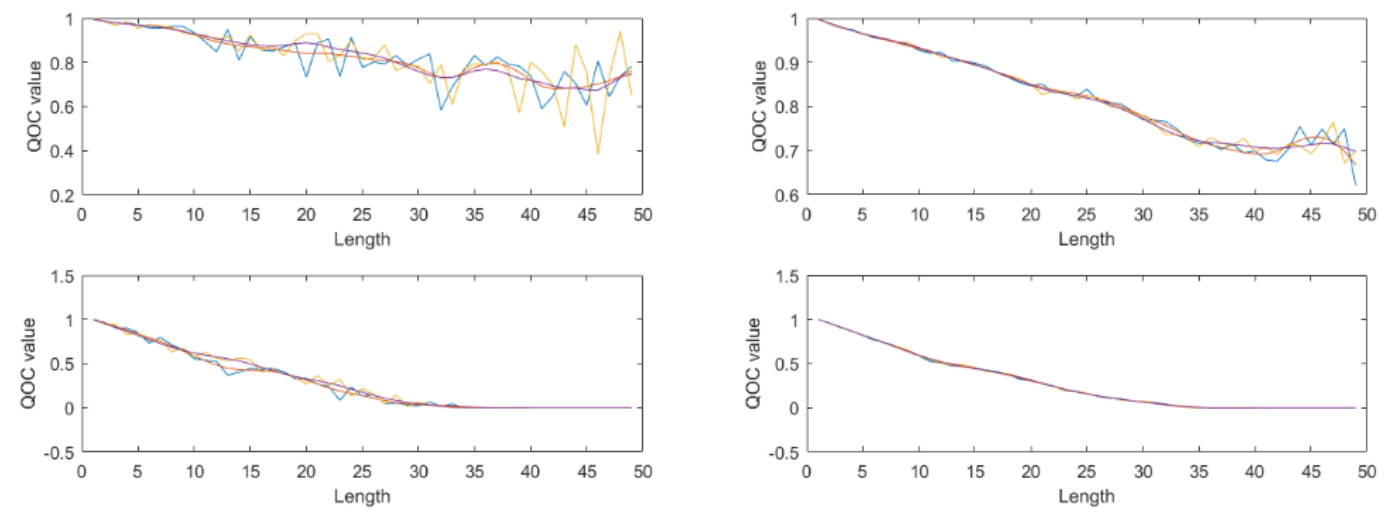

(a)

(b)

Fig. 9. QCF with random number of: a) 100, b) 500

3. To make the calculation more accurate, images were chosen from sections in all three main directions. The QCF functions of reconstructed samples with different $\alpha$ from Eq. (2.13) are compared with QCF functions of the real bone with the same volume fraction. Error is defined by Eq. (3.2).

$$
\text { Error }=\frac{1}{m} \sum_{s=0}^{s=50} \frac{\left|Q C F(s)_{f_{\text {inal_reconstructed_model }}-Q C F(s)_{\text {novel_model }} \mid}\right|}{Q C F(s)_{\text {novel_model }}}
$$

Where $\mathrm{m}$ is the number of sections analyzed and $\mathrm{s}$ is the size of vectors. According to our aforementioned assumption, for our study $\mathrm{m}$ would be 50 where $\mathrm{m}=1: 16,17: 33$ and $34: 50$ refers to $\mathrm{X}, \mathrm{Y}$ and $\mathrm{Z}$ directions, respectively.

Errors are computed for each $\alpha$ in the 31,41 and $44 \%$ volume fraction for each direction separately as like as it is shown in Table 5 . 
Table 5. QCF errors for reconstructed microstructures with $41 \%$ volume fraction.

\begin{tabular}{cccccc}
\hline $\boldsymbol{\alpha}$ & Model Number & Horizontal & Vertical & Total & Rank \\
\hline $\mathbf{0 . 1}$ & 1 & 0.0737 & 0.0846 & 0.0800 & 4 \\
\hline $\mathbf{0 . 2}$ & 2 & 0.0606 & 0.0886 & 0.0746 & 3 \\
\hline $\mathbf{0 . 3}$ & 3 & 0.0785 & 0.0903 & 0.0844 & 7 \\
\hline $\mathbf{0 . 4}$ & 4 & 0.0496 & 0.0893 & 0.0694 & 1 \\
\hline $\mathbf{0 . 5}$ & 5 & 0.0599 & 0.0868 & 0.0733 & 2 \\
\hline $\mathbf{0 . 6}$ & 6 & 0.0714 & 0.0926 & 0.0820 & 5 \\
\hline $\mathbf{0 . 7}$ & 7 & 0.0697 & 0.0989 & 0.0843 & 6 \\
\hline $\mathbf{0 . 8}$ & 8 & 0.0794 & 0.0984 & 0.0986 & 10 \\
\hline $\mathbf{0 . 9}$ & 9 & 0.0848 & 0.1038 & 0.0943 & 9 \\
\hline
\end{tabular}

The decision is made based on the average of three directions similarly to Table 6 below.

Table 6. QCF errors for reconstructed matrices with $41 \%$ volume fraction in three main directions separately.

\begin{tabular}{cccccccc}
\hline Direction & \multicolumn{3}{c}{ Horizontal } & \multicolumn{4}{c}{ Vertical } \\
\hline Plane & XY & XZ & YZ & XY & XZ & YZ \\
\hline $\mathbf{1}$ & 0.0880 & 0.0370 & 0.0963 & 0.0540 & 0.0972 & 0.1026 \\
\hline $\mathbf{2}$ & 0.0603 & 0.0331 & 0.0886 & 0.0534 & 0.0865 & 0.1259 \\
\hline $\mathbf{3}$ & 0.1092 & 0.0340 & 0.0924 & 0.0463 & 0.1175 & 0.1071 \\
\hline $\mathbf{4}$ & 0.0339 & 0.0326 & 0.0825 & 0.0629 & 0.0926 & 0.1126 \\
\hline $\mathbf{5}$ & 0.0698 & 0.0315 & 0.0786 & 0.0632 & 0.1003 & 0.0969 \\
\hline $\mathbf{6}$ & 0.0818 & 0.0307 & 0.1018 & 0.0582 & 0.1018 & 0.1179 \\
\hline $\mathbf{7}$ & 0.0649 & 0.0389 & 0.1053 & 0.0495 & 0.1240 & 0.1232 \\
\hline $\mathbf{8}$ & 0.0889 & 0.0582 & 0.0912 & 0.0701 & 0.0939 & 0.1205 \\
\hline $\mathbf{9}$ & 0.1103 & 0.0277 & 0.1164 & 0.0595 & 0.1224 & 0.1295 \\
\hline $\mathbf{1 0}$ & 0.0819 & 0.0354 & 0.1361 & 0.0556 & 0.1028 & 0.1284 \\
\hline
\end{tabular}

As it is shown in Table 5, $\alpha=0.4$ gives the best result and $\alpha=0.8$ has the highest value of error. In the previous method, $\alpha=0.4$ gave the best result $\alpha=1$ had the highest value of error. By calculating their mechanical characterization, they could be compared. In Table 7, the best and worst coefficients of other volume fractions are provided.

Table 7. The best and the worst coefficients for each volume fraction via QCF method.

\begin{tabular}{ccccc}
\hline & best $\boldsymbol{\alpha}$ & Error (\%) & worst $\boldsymbol{\alpha}$ & Error (\%) \\
\hline $\mathbf{3 1 \%}$ & 0.4 & 6.94 & 0.8 & 9.86 \\
\hline $\mathbf{4 1 \%}$ & 0.1 & 6.00 & 0.8 & 8.03 \\
\hline $\mathbf{4 4 \%}$ & 0.1 & 7.30 & 0.5 & 9.97 \\
\hline
\end{tabular}

Of course, porosities are 3D in space and this was analyzed in this section. However, although choosing 3D vectors would be more accurate, it would also be more time consuming and complicated to implement. This can be examined at a later stage in future studies.

\subsection{Mechanical Characterization}

Of course, bone is a living material and the couplings between biology and mechanics are important factors in the determination of its evolution as a function of time, particularly for ageing people developing osteoporosis. This was described partly in the Introduction section. However, in order to build precise predictive numerical model, being if possible patient dependent, one need to account for the distribution of bone microstructure in the 
model that will lead to the patient variability. Unfortunately, it is still not easy to build one microstructure bone model per one patient each time. This is far too time consuming and not relevant for the current knowledge state (most of the bone mecanobiological parameters are still unknown for long time prediction). Hence, improved methods that are able to account for different types of bone microstructures leading to patient variability are desirable. Statistical reconstruction is a powerful tool that is able to provide insight of these different microstructures effects for long term evolution. However, the specificity of bone microstructure (namely clustering and microstructure interconnections) needs to be implemented specifically within these statistical tools. To validate the models generated from Two-phase recovery method and optimized via statistical descriptors of TPCF and QCF, their mechanical properties (effective elastic modulus in the main direction) were compared with the models with the same volume fractions of [Bagherian et al. 2019] models that are named original bone in the Table 8 .

It is presumed that bone microstructures are formed on two phases including hard and soft matters and having linear elastic mechanical behavior. The average value of Young modulus determined for hard and soft phases are $15 \mathrm{GPa}$ and $1 \mathrm{KPa}$, in order. In addition, Poisson's ratio for both phases is 0.3 .

Based on the method that was explained in section 2.3, the mechanical properties of the selected reconstructed models and real bone models, Young modulus in the Haversian direction, where the bone has the highest strength, are calculated. The outcomes of this estimation are brought in the table below.

Table 8. The Young modulus for each microstructure.

\begin{tabular}{|c|c|c|}
\hline Model & Effective Elastic Modulus (Pa & Note \\
\hline $23 \%$ Original Bone & $9.4498 \mathrm{e}+08$ & \\
\hline $31 \%$ Original Bone & $2.2583 \mathrm{e}+09$ & \\
\hline $31 \%$ Best QCF & $1.8875 \mathrm{e}+09$ & 16 percent \\
\hline $31 \%$ Best TPCF & $1.8356 \mathrm{e}+09$ & Slight Difference with QCF \\
\hline 31\% Worst QCF & $1.4773 \mathrm{e}+09$ & 34 percent \\
\hline $31 \%$ Worst TPCF & $1.5173 \mathrm{e}+09$ & Slight Difference with QCF \\
\hline $41 \%$ Original Bone & $4.3750 \mathrm{e}+09$ & \\
\hline $41 \%$ Best QCF & $3.5338 \mathrm{e}+09$ & 19 percent \\
\hline $41 \%$ Best TPCF & $3.5338 \mathrm{e}+09$ & Same as QCF \\
\hline $41 \%$ Worst QCF & $2.6648 \mathrm{e}+09$ & 39 percent \\
\hline $41 \%$ Worst TPCF & $2.8848 \mathrm{e}+09$ & Acceptable Difference with QCI \\
\hline $43 \%$ Original Bone & $5.1634 \mathrm{e}+09$ & \\
\hline $43 \%$ Best QCF & $3.9572 \mathrm{e}+09$ & 23 percent \\
\hline 43\% Best TPCF & $3.9572 \mathrm{e}+09$ & Same as QCF \\
\hline 43\% Worst QCF & $2.8680 \mathrm{e}+09$ & 44 percent \\
\hline $43 \%$ Worst TPCF & $3.2863 \mathrm{e}+09$ & \\
\hline $47 \%$ Original Bone & $5.9400 \mathrm{e}+09$ & \\
\hline
\end{tabular}

The Young modulus values in the table are extracted from the homogenized constitutive matrix. For instance, $\mathrm{C}^{\mathrm{H}}$ of the $41 \%$ Best QCF is:

$$
\left[\begin{array}{cccccc}
0.1537 * e-08 & -0.0072 * e-08 & -0.0220 * e-08 & -0.0089 * e-08 & 0.0022 * e-08 & 0.0378 * e-08 \\
-0.072 * e-08 & 0.0283 * e-08 & -0.0091 * e-08 & -0.0046 * e-08 & 0.0018 * e-08 & 0.0004 * e-08 \\
-0.0220 * e-08 & -0.0091 * e-08 & 0.2228 * e-08 & 0.0018 * e-08 & 0.0037 * e-08 & 0.0082 * e-08 \\
-0.0089 * e-08 & -0.0046 * e-08 & 0.0018 * e-08 & 0.2598 * e-08 & 0.0227 * e-08 & 0.0073 * e-08 \\
0.0022 * e-08 & 0.0018 * e-08 & 0.0037 * e-08 & 0.0227 * e-08 & 0.3087 * e-08 & -0.0202 * e-08 \\
0.0378 * e-08 & 0.0004 * e-08 & 0.0082 * e-08 & 0.0073 * e-08 & -0.0202 * e-08 & 0.7870 * e-08
\end{array}\right]
$$

The inverse value of arrays $[1,1]$ and $[2,2]$ in the effective compliance matrix are equal to the Young modulus in $(1,0,0)$ and $(0,1,0)$ directions, respectively. Hence, they are 0.650 and $3.533 \mathrm{GPa}$ in order. Not only does this result prove that bone is stronger in the Haversian direction, but it also demonstrates a contradiction between Young modulus of random matrices and reconstructed ones. The differences between the mechanical properties of reconstructed and the real bone structures, which is between 16 and $23 \%$, beside the morphology resemblances clarifies the validity of the method according to [Bagherian et al. 2019]. On the one hand, results verify that TPCF is capable enough to predict most of the suitable models in the interpolation method; but on the other hand, QCF is more specific in all cases thanks to the addition of the connections. For example, in the $31 \%$ samples, QCF anticipated the better model and in all three cases, it also predicted the worse models. 


\subsection{Volume Rendering of Reconstructed Models}

One difficulty in the the prediction of bone density evolution is the importance of the distribution of microstructure as it impacts importantly the long term effects. Two of the best reconstructed samples are presented here. As shown on figure 10, the overall morphologies are saved compared to random matrices.
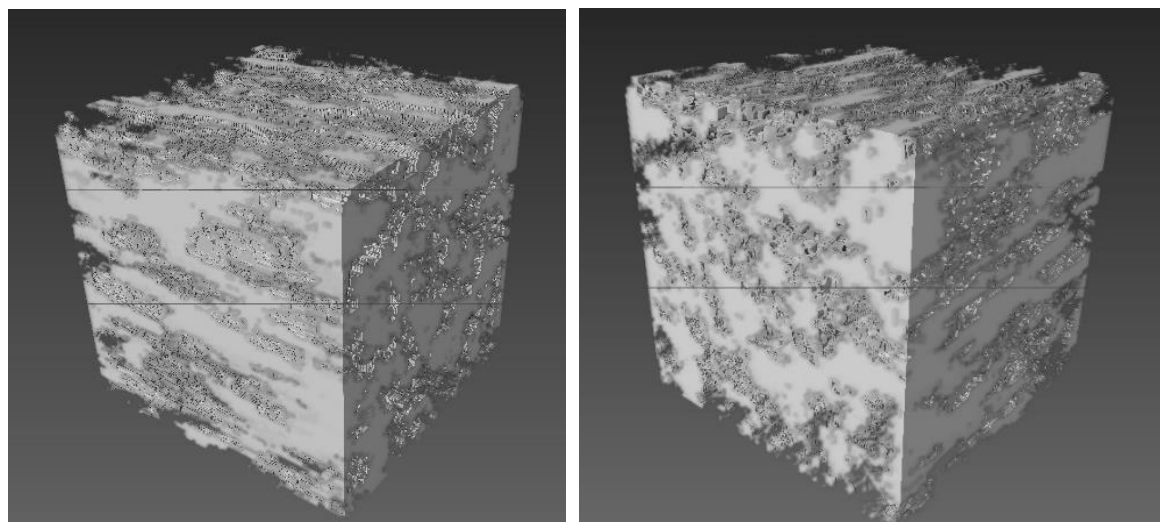

(a)
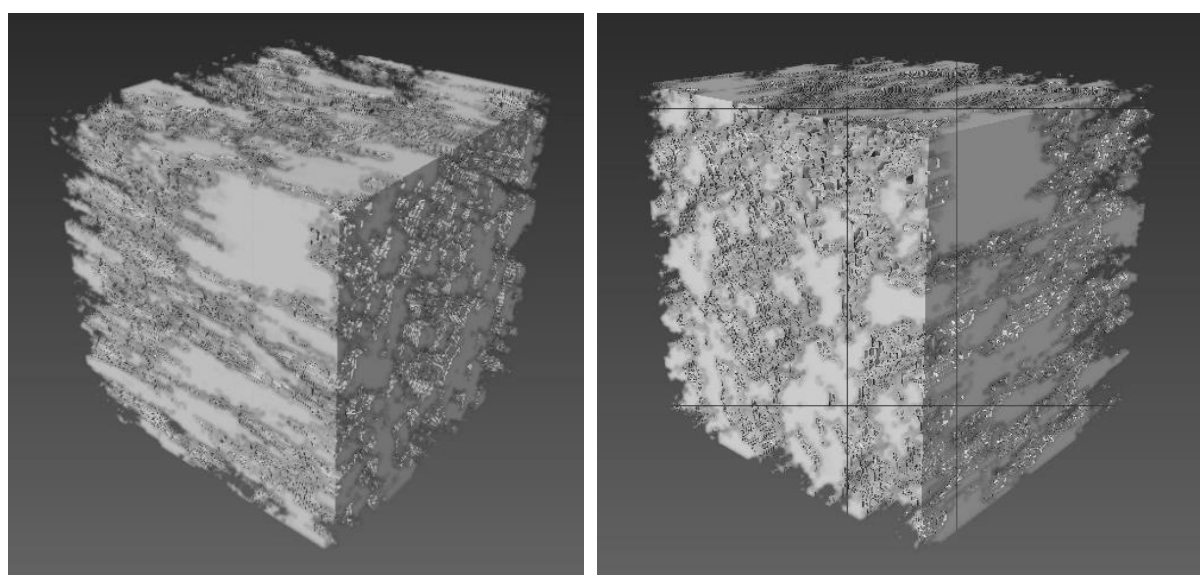

(b)

Fig. 10. The Best reconstructed images from two-phase recovery of samples with volume fraction of: a) $41 \%$, b) $31 \%$.

The statistical method proves to be a useful tool for developing specific material microstructure distribution. However, the TPCF fell short in providing adequate reconstruction due to the specific bone microstructure that needs to account for phase connectivity and clustering. Hence a new descriptor called QCF (Quality of Connection Function) was used to improve this. It showed improvement over TPCF and its ability to provide better bone microstructure distribution as presented in Figure 10. Since there is currently no equivalent work available in the literature, this constitutes a first approach and should be completed with future studies. However, it showed the possibility to predict with good accuracy the variability of bone microstructure and therefore possible patient dependent studies. This method can also be used for other materials presenting similar characteristics.

\section{Conclusion}

In this study, the cancellous bone microstructures were studied. At first, the primary structure was degraded with one of the most novel methods to reach the lowest volume fraction; this procedure was used instead of the real process of osteoporosis which is pertained to the real patient. Then, the interpolated volume fractions were gained from the highest and lowest models based on reconstruction methodology. Each microstructure with the specific volume fraction was optimized through comparing TPCF and QCF amounts of the reconstructed ones with the real one at the same volume fraction (We were looking for the optimized and repeatable Alfa in each step). In the last step, the models were verified by comparing their Young Modulus, and also the advantage of 
our procedure was evaluated. The reconstructed models have a TPCF error under one percent by contrast with the randomized matrices having about 10-20 percent. This would cause geometries resemblance and similar mechanical properties among reconstructed models and the real bone structures. Moreover, the optimization method has improved the effective elastic modulus similarity between reconstructed models and the real bone. The effective elastic modulus differences between the worst and the best estimation are 18, 20 and 21 percent for 31,41 and $43 \%$ reconstructed models, respectively. According to the results discussed, the following conclusion can be made:

- QCF function based on 2D vectors can be applied for 3D microstructures via the step by step innovation presented in the results section instead of computing QCF with 3D vectors.

- Interpolation with the reconstruction aforesaid method is an appropriate procedure for estimating the morphologies.

- By computing QCF in the reconstructed models, the detection of best and worst Alfa was improved according to the TPCF detector.

- According to the Young Modulus results, it is shown that TPCF is still capable of detecting the best microstructure.

Although QCF was calculated using 2D vectors because of time-consuming calculations, it showed reasonable results. In future works, QCF with 3D vectors can be studied. Furthermore, a reconstruction based on QCF function can be generated to improve the models accuracy. Finally, although we have simplified the bone model to propose the application of statistical functions in bone structures, more complicated biological structures can be studied in the future using this approach.

\section{References}

\section{Journal references:}

Abali, B. E., et al. (2017). "Theory and computation of higher gradient elasticity theories based on action principles." Archive of Applied Mechanics 87(9): 1495-1510.

Andreassen, E. and C. S. Andreasen (2014). "How to determine composite material properties using numerical homogenization." Computational Materials Science 83: 488-495.

Andreaus, U., et al. (2011). "Optimal-tuning PID control of adaptive materials for structural efficiency." Structural and Multidisciplinary Optimization 43(1): 43-59.

Bagherian, A., et al. (2019). "A novel numerical model for the prediction of patient-dependent bone density loss in microgravity based on micro-CT images." Continuum Mechanics and Thermodynamics: 1-17.

Bakalova, L., et al. (2018). "Relating intracortical bone mechanics to pore morphology and remodeling characteristics in the human fibula." J. Bone Miner. Res.

Baniassadi, M., et al. (2011). "Three-phase solid oxide fuel cell anode microstructure realization using two-point correlation functions." Acta materialia 59(1): 30-43.

Casanova, R., et al. (2010). "Temporal evolution of skeletal regenerated tissue: what can mechanical investigation add to biological?" Medical \& biological engineering \& computing 48(8): 811-819.

Chen, H., et al. (2013). "Age-related changes in trabecular and cortical bone microstructure." International journal of endocrinology 2013.

Dell'Isola, F., et al. (2015). "At the origins and in the vanguard of peridynamics, non-local and higher-gradient continuum mechanics: an underestimated and still topical contribution of Gabrio Piola." Mathematics and Mechanics of Solids 20(8): 887-928.

Dell'Isola, F., et al. (2017). "Higher-gradient continua: The legacy of Piola, Mindlin, Sedov and Toupin and some future research perspectives." Mathematics and Mechanics of Solids 22(4): 852-872.

Doblaré, M. and J. Garcia (2002). "Anisotropic bone remodelling model based on a continuum damage-repair theory." Journal of biomechanics 35(1): 1-17.

Fullwood, D. T., et al. (2008). "A strong contrast homogenization formulation for multi-phase anisotropic materials." Journal of the Mechanics and Physics of Solids 56(6): 2287-2297.

George et al. (2017). "Examples of multiscale and multiphysics numerical modeling of biological tissues." Biomedical materials and engineering 28(s1): S15-S27. 
Ghimire, S., et al. (2018). "Role of dynamic loading on early stage of bone fracture healing." Annals of biomedical engineering 46(11), 1768-1784.

Hasanabadi, A., et al. (2016). "3D microstructural reconstruction of heterogeneous materials from 2D cross sections: a modified phase-recovery algorithm." Computational Materials Science 111: 107-115.

Hashemi, M. S., et al. (2019). "A novel machine learning based computational framework for homogenization of heterogeneous soft materials: application to liver tissue." Biomechanics and modeling in Mechanobiology: $1-12$.

Hegedus, D. and S. Cowin (1976). "Bone remodeling II: small strain adaptive elasticity." Journal of elasticity 6(4): 337-352.

Hollister, S. J. (2005). "Porous scaffold design for tissue engineering." Nature materials 4(7): 518-524.

Horvath, A. L. (2006). "Solubility of structurally complicated materials: II. Bone." Journal of physical and chemical reference data 35(4): 1653-1668.

Huiskes, R., et al. (2000). "Effects of mechanical forces on maintenance and adaptation of form in trabecular bone." Nature 405(6787): 704-706.

Izadi, H., et al. (2017). "Application of full set of two point correlation functions from a pair of 2D cut sections for 3D porous media reconstruction." Journal of Petroleum Science and Engineering 149: 789-800.

Kazempour, M., et al. (2019). "Homogenization of heterogeneous brain tissue under quasi-static loading: a visco-hyperelastic model of a 3D RVE." Biomechanics and modeling in Mechanobiology 18(4): 969-981.

Kazempour, M., et al. (2019). "Numerical Simulation of Osteoporosis Degradation at Local Scale: A Preliminary Study on the Kinematic Loss of Mechanical Bone Stiffness and Microstructure." Stem Cells and Regenerative Medicine Proceedings of the $8^{\text {th }}$ International China-Europe Symposium, Wuhan, China, June 2018, (IOS Press).

Kraiem, T., et al. (2020). "Computational approach of the cortical bone mechanical behavior based on an elastic viscoplastic damageable constitutive model," International Journal of Applied Mechanics 12(07), 2050081.

Lang, T. F., et al. (2006). "Adaptation of the proximal femur to skeletal reloading after long-duration spaceflight." Journal of Bone and Mineral Research 21(8): 1224-1230.

Lekszycki, T. (2002). "Modelling of bone adaptation based on an optimal response hypothesis." Meccanica 37(45): 343-354

Lekszycki, T. (2005). "Functional adaptation of bone as an optimal control problem." Journal of Theoretical and Applied Mechanics 43(3): 555-574.

Madeo, A., et al. (2012). "A second gradient continuum model accounting for some effects of micro-structure on reconstructed bone remodelling." Comptes Rendus Mécanique 340(8): 575-589

Martin, M., et al. (2017). "A thermodynamically consistent model of bone rotary remodeling: a 2D study." Computer methods in biomechanics and biomedical engineering 20(sup1): 127-128.

Misra, A. and P. Poorsolhjouy (2015). "Identification of higher-order elastic constants for grain assemblies based upon granular micromechanics." Mathematics and Mechanics of Complex Systems 3(3): 285-308.

Pivonka, P., et al. (2008). "Model structure and control of bone remodeling: a theoretical study." Bone 43(2): 249-263.

Placidi, L., et al. (2015). "Gedanken experiments for the determination of two-dimensional linear second gradient elasticity coefficients." Zeitschrift für angewandte Mathematik und Physik 66(6): 3699-3725.

Prendergast, P. and D. Taylor (1994). "Prediction of bone adaptation using damage accumulation." Journal of biomechanics 27(8): 1067-1076.

Ram, R. R. (2006). "HIERARCHY OF BONE STRUCTURE REPORT."

Rémond, Y., et al. (2016). Applied RVE reconstruction and homogenization of heterogeneous materials, Wiley Online Library.

Scala, I., et al. (2017). "Mechanically-driven bone remodeling simulation: Application to LIPUS treated rat calvarial defects." Mathematics and Mechanics of Solids 22(10): 1976-1988.

Sheidaei, A., et al. (2019). "Influence of bone microstructure distribution on developed mechanical energy for bone remodeling using a statistical reconstruction method." Mathematics and Mechanics of Solids 24(10): 3027-3041.

Spingarn, C., et al. (2017). "Multiphysics of bone remodeling: a 2D mesoscale activation simulation." Biomedical materials and engineering 28(s1): S153-S158. 
Tawara, D. et al. (2019). "Prediction of Bone Quality of Remodeling Trabeculae Using Multi-Scale Stress Analyses with a Homogenization Technique Reflecting Material Anisotropy, " International Journal of Applied Mechanics 11(06), 1950055.

Torquato, S. and H. Haslach Jr (2002). "Random heterogeneous materials: microstructure and macroscopic properties." Appl. Mech. Rev. 55(4): B62-B63.

\section{Book reference}

\section{Proceedings reference:}

Carter, D., et al. (1996). "Mechanical factors in bone growth and development." Bone 18(1): S5-S10.

Chiba, K., et al. (2013). "Heterogeneity of bone microstructure in the femoral head in patients with osteoporosis: an ex vivo HR-pQCT study." Bone 56(1): 139-146. 